Cite this: Soft Matter, 2014, 10, 1309

\title{
Tunable shape transformation of freezing liquid water marbles
}

Received 10th July 2013

Duyang Zang, ${ }^{\text {a }}$ Kejun Lin, ${ }^{a}$ Wenkai Wang, ${ }^{a}$ Yaxi Gu, ${ }^{a}$ Yongjian Zhang, ${ }^{b}$ Xingguo Geng ${ }^{a}$ and Bernard P. Binks*c

Accepted 23rd August 2013

DOI: $10.1039 / c 3 s m 51885 j$

www.rsc.org/softmatter

Liquid water marbles coated with fumed silica nanoparticles exhibit various shape transformations upon freezing which are dependent on the hydrophobicity of the nanoparticles. The shape can be recovered during re-melting. For marbles coated with the most hydrophobic particles, a vertically prolonged morphology with a pointed protrusion on the top is formed on freezing. For marbles coated with less hydrophobic particles, a lateral expanded flying saucer-shaped morphology is formed. The different responses to freezing result from the different heterogeneous nucleation sites owing to the different positions of the particles at the air-water interface. If the particles are more immersed in water, ice embryos tend to form in the concave cavities between the particles. The volume expansion of water caused by freezing and continuous nucleation lead to continuous lateral stretching of the particle network coating the droplet surface and ultimately to the horizontally inflated shape of the marble. If the particles are more exposed to air, nucleation occurs on the convex surface of the particles, similar to that of a bare water droplet on a hydrophobic substrate.

Liquid marbles, constituted by a liquid core and a solid shell formed by the adsorption of micro/nanometer sized particles on the droplet-vapor interface, have attracted increasing research interest in recent years. ${ }^{1-5}$ The particle shell prevents direct contact of the liquid with a solid surface, thus reducing the friction between the droplet and the substrate. ${ }^{6}$ Furthermore, liquid marbles often exhibit remarkable mechanical robustness towards compression due to the elastic particulate thin film. ${ }^{7}$ Liquid marbles are thus excellent candidates for application as micro-reservoirs, which can be easily transported without any leakage of the encapsulated liquid. Owing to this advantage,

${ }^{a}$ Key Laboratory of Space Applied Physics and Chemistry of Ministry of Education, School of Science, Northwestern Polytechnical University, Xi'an, 710129, China. E-mail:dyzang@nwpu.edu.cn; Tel: +86 29-88431618

${ }^{b}$ State Key Laboratory of Solidification Processing, School of Materials Science and Engineering, Northwestern Polytechnical University, Xi'an 710072, China

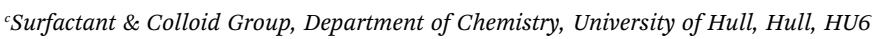
7RX, UK. E-mail: b.p.binks@hull.ac.uk; Tel: +44 (0)1482-465450 they have great potential in the applications of microfluidics ${ }^{8}$ and micro-reactors. ${ }^{9-11}$ In addition to their non-stick behavior and mechanical robustness, liquid marbles respond to different external fields. ${ }^{11-13}$ When exposed to an electric field, the shape of the marble is characterized as a prolate spheroid with the eccentricity increasing non-linearly with the electric field strength, ${ }^{\mathbf{1 2}}$ unlike the case of a bare droplet where a linear dependence of eccentricity occurs. ${ }^{14}$ It has also been reported that liquid marbles can be actuated with an electric field, resulting in the climbing motion of the marbles. ${ }^{15}$ If the liquid marbles are coated with hydrophobic ferromagnetic particles such as $\mathrm{Fe}_{3} \mathrm{O}_{4}$, the open/close switch of liquid marbles can be effected with a magnetic field. ${ }^{9}$

In addition to electric and magnetic fields, liquid marbles can respond to the temperature. Recently, it was reported that liquid water marbles coated with lycopodium particles underwent a unique shape transition from spherical to a flying saucer-shaped morphology upon freezing. ${ }^{16}$ This is in contrast to the behavior observed for a bare water droplet (no coating) in which no sideways expansion was observed. Hashmi et al. attributed the phenomenon observed with liquid marbles to the preferred nucleation induced by solid particles and Marangoni convection. ${ }^{\mathbf{1 6}}$ The striking shape transition of a liquid marble during freezing suggests that the coating of particles on the droplet surface may drastically alter its response to the environment. However, a natural question arises as whether and how the particle hydrophobicity influences the shape transition upon freezing.

In this work, we prepare liquid water marbles coated with fumed silica nanoparticles of various hydrophobicities and investigate their shape evolution upon freezing. The silica particles were provided by Wacker-Chemie (Germany) and were used as received. The surfaces of the particles are modified by reaction of the silanol groups with dichlorodimethylsilane to obtain particles with different hydrophobicities. In this study, we used particles with a relative SiOH content between 14 and $61 \%$, in which the hydrophobicity increases with decreasing $\mathrm{SiOH}$ content. The primary particles are quasi-spherical of approximately $20 \mathrm{~nm}$ in diameter. The water used in the 
experiments was purified with an Ultrapure Water System (EPED, China). The freezing experiments of the marbles were performed at a relative humidity of approx. $40 \%$. The preparation details of the marbles can be found elsewhere. ${ }^{17}$ The volumes of the liquid water marbles were 15 and $20 \mu \mathrm{L}$. To observe the shape evolution upon freezing and re-melting, the marble was placed directly on a thermo-electric cooler plate (TEC1-12706, Xinghe Electro, China) held at a temperature of $\sim-15{ }^{\circ} \mathrm{C}$. For the freezing experiments of a bare water droplet, the droplet was placed on a super-hydrophobic substrate, fabricated by a chemical deposition method, ${ }^{18}$ which was placed on top of the cooler plate. A high speed camera (Allied, Pike F-032, $200 \mathrm{fps}$ ) was used to monitor the side view of the marbles. The image data were analyzed using MaxTRAQ software. Advancing contact angles of water and ice in air on silica substrates were measured by depositing and freezing a water droplet $(2 \mu \mathrm{L})$ on a flat disc composed of the fumed silica powders. The silica disc was formed by pressing the silica powder at up to $26 \mathrm{MPa}$ in an oil hydraulic press. The contact angles of the droplet just before and after freezing (at $\sim-5{ }^{\circ} \mathrm{C}$ ) were recorded using the sessile drop method on a contact angle measurement apparatus (Powereach, China).

Fig. 1 shows the illustration of the final shapes of a bare water droplet and nanoparticle-coated liquid water marbles after solidification on the cold plate. It is clear that the final shapes of the marbles strongly depend on the hydrophobicity of the particles coating them. It should be noted, as will be addressed in Fig. 2, that the shapes of the water droplet and water marbles evolve during the freezing process rather than forming immediately. The varied final shapes are not determined by their initial shapes (contact angles) because all of them exhibit a similar spherical initial shape on the substrate. For the marbles coated by the most hydrophobic silica particles, 14\% SiOH (Fig. 1(b)), it became prolonged in the vertical direction and a pointed protrusion was observed on the top, which is similar to what is observed for a bare water droplet, Fig. 1(a). The protrusion is mainly due to the volume expansion of water on freezing. ${ }^{19,20}$ In addition, the top protrusion also appears upon freezing of a pure water droplet deposited on a hydrophilic substrate where the droplet presents an initial morphology of a spherical cap, ${ }^{\mathbf{2 0 , 2 1}}$ confirming that the initial shape is not an essential factor for the formation of a protrusion. With decreasing particle hydrophobicity, the vertical prolonged effect becomes weak (Fig. 1(c)). For particles of intermediate hydrophobicity (Fig. 1(d)), the marble retains its original spherical shape. With a further decrease in

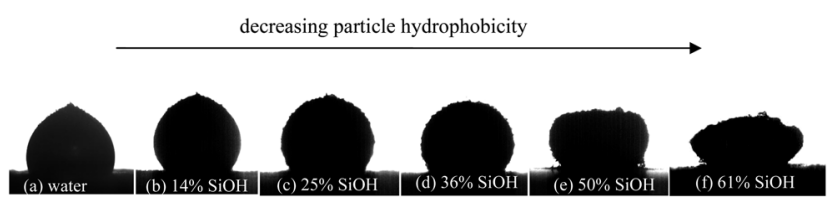

Fig. 1 Final shapes of a solidified bare water droplet and liquid marbles (90 s since being placed on a cold plate) from vertically prolonged morphology with a pointed protrusion via spherical shape to a flying saucer-shaped morphology. The changes occur on decreasing the particle hydrophobicity, quantified by the $\mathrm{SiOH}$ content on their surfaces. The volume of original marbles is $15 \mu \mathrm{L}$. particle hydrophobicity, the marble starts to inflate in the horizontal direction, i.e. its height decreases, Fig. 1(e). Finally, for the least hydrophobic particles, 61\% $\mathrm{SiOH}$ (Fig. 1(f)), the frozen marble exhibits a flying saucer-shaped morphology, as reported by Hashmi et al. for one particle type. ${ }^{16}$ In addition to the existing study, our results demonstrate that various shapes of frozen marbles can be obtained by tuning the particle hydrophobicity.

To further study the shape evolution of the marbles, a series of images during cooling and heating was recorded using a highspeed camera, as shown in Fig. 2. During cooling, the marbles start to freeze from the bottom which is in contact with the cold plate, suggesting that freezing takes place via heterogeneous nucleation. For the bare water droplet, the substrate which supports the droplet acts as the nucleation site. For the liquid marbles however, the particles coating the droplet surface act as nucleation sites. In all cases, particles are visible completely covering the marble surface after freezing, i.e. no lateral displacement occurs. However, it should be noted that the lowest temperature at the marble bottom favours overcoming of the nucleation barrier on the particle surfaces. The ice-water interface (see arrows) propagates upwards until the marble is completely solidified, Fig. 2(e). This is similar to what is observed with a pure water droplet freezing on a copper substrate which is partially hydrophilic. ${ }^{19}$ Continuous cooling leads to the formation of frost on top of the marbles (Fig. 2(f)), developing mostly on marbles coated with the least hydrophobic particles (Fig. 2(f), $61 \% \mathrm{SiOH}$ ). Frost is the solid deposition of water vapour from humid air. For all the particle types, the deformed marble caused by freezing recovers its original shape upon heating, Fig. $2(\mathrm{~g})-(\mathrm{j})$. However, for marbles coated with the least hydrophobic particles $(50 \%$ and $61 \% \mathrm{SiOH})$, their final heights are slightly lower than those before freezing probably due to leakage of water during remelting. This may be because for relatively hydrophilic particles some ice crystals may penetrate the particle layer and consequently water comes in direct contact with the substrate upon remelting. For the marbles coated with the most hydrophobic particles (Fig. 2, 14\% SiOH), their contact area with the substrate always remains constant. The surface curvature does not change in the early stage of freezing. The vertical elongation and pointed protrusion appear at the later stages of freezing. However, for marbles coated with less hydrophobic particles, the contact area increases at first and subsequently the marble starts to inflate in the horizontal direction.

To investigate whether the shape change depends on the temperature gradient, the liquid marbles were placed in a freezer and held at a temperature of $\sim-15{ }^{\circ} \mathrm{C}$, i.e. they were in a more uniform external temperature field. In this case, the temperature gradient inside the droplet was greatly reduced. However, similar shape changes were observed as reported above: prolonged shape for marbles coated with very hydrophobic particles, Fig. 3(a) and (b), and flattened shape for marbles coated with less hydrophobic particles, Fig. 3(c) and (d). The results suggest that the temperature gradient may not be an important factor in the shape changes occurring during the freezing of liquid marbles. As a consequence, the argument put forward concerning the Marangoni convection induced by such a temperature gradient ${ }^{\mathbf{1 6}}$ may not be applicable here. 


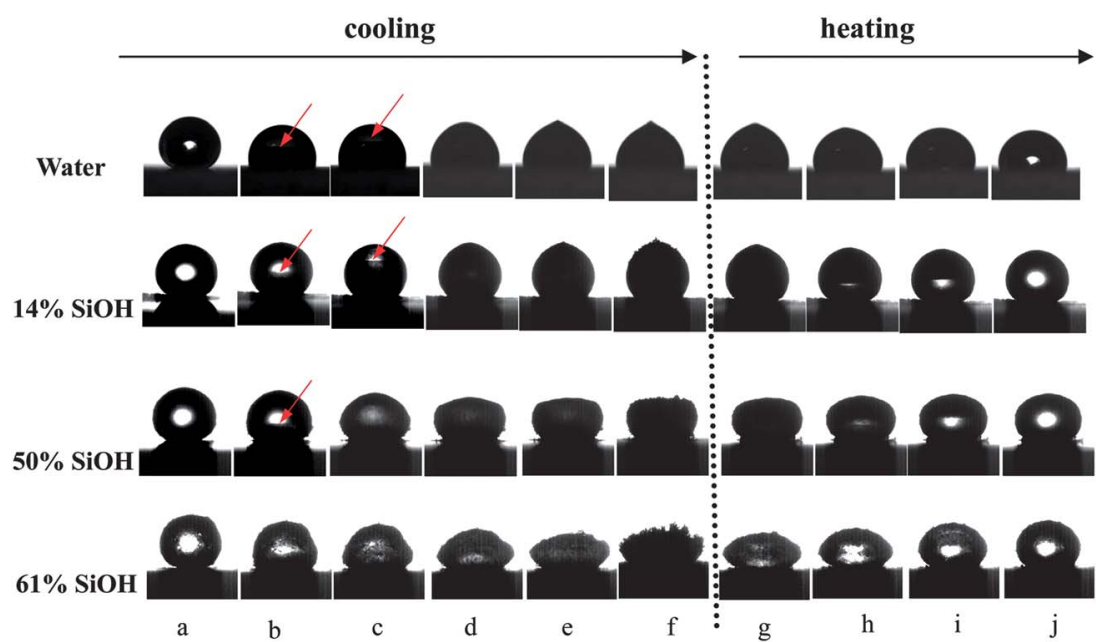

Fig. 2 Shape evolution of a bare water droplet and liquid marbles $(15 \mu \mathrm{L})$ upon freezing $(a-f)$ and re-melting $(g-j)$. The marbles are coated with silica particles of different hydrophobicities (\% SiOH). The arrows mark the advancing ice-water interface upwards. The time scales involved in freezing and re-melting are $\sim 90 \mathrm{~s}$.

From the above results, a natural question arises: why do the marbles coated with the very hydrophobic and least hydrophobic particles show a very different response upon freezing? The underlying reason may be the different nucleation mechanisms induced by the particles which act as nuclei for freezing.

The catalytic effect of a planar substrate on heterogeneous nucleation can reduce the free energy barrier for nucleation: ${ }^{22}$

$$
\begin{gathered}
\Delta G_{\mathrm{he}}^{*}=\frac{16 \pi \sigma_{\mathrm{LS}}^{3}}{3 \Delta G_{\mathrm{m}}{ }^{2}}\left(\frac{2-3 \cos \theta_{\mathrm{n}}+\cos ^{3} \theta_{\mathrm{n}}}{4}\right)=\Delta G_{\mathrm{ho}}^{*} f\left(\theta_{\mathrm{n}}\right) \\
f\left(\theta_{\mathrm{n}}\right)=\frac{1}{4}\left(2-3 \cos \theta_{\mathrm{n}}+\cos ^{3} \theta_{\mathrm{n}}\right)
\end{gathered}
$$

where $\Delta G_{\text {he }}^{*}$ and $\Delta G_{\text {ho }}^{*}$ are the free energies associated with formation of a nucleus of the critical radius in heterogeneous and homogeneous nucleation respectively, $\sigma_{\mathrm{LS}}$ is the liquid (water)solid (ice) interfacial energy, $\Delta G_{\mathrm{m}}$ is the Gibbs free energy difference between the parent phase (water) and the new phase which forms (ice) per unit volume and $\theta_{\mathrm{n}}$ is the contact angle of the newly emerging phase (ice) in water with the substrate (particles in the present study), measured through ice. The function $f\left(\theta_{\mathrm{n}}\right), 0 \leq f\left(\theta_{\mathrm{n}}\right) \leq 1$, represents the ratio of $\Delta G_{\mathrm{he}}^{*} / \Delta G_{\mathrm{ho}}^{*}$.

As shown in eqn (1) and (2), $\theta_{\mathrm{n}}$ plays an important role in the nucleation process. However, it is currently quite a challenge to obtain the exact value of $\theta_{\mathrm{n}}$ due to the difficulty in obtaining the interfacial energies $\sigma_{\text {silica/water }}$ and $\sigma_{\text {silica/ice }}$. To make a reasonable estimation, we proceed as follows. Based on Young's equations relating to 'drops' of ice in either water or air and a water droplet in air on silica substrates, the corresponding contact angles $\theta_{\mathrm{n}}, \theta_{\text {ice/air }}$ and $\theta_{\text {water/air }}$ on a silica substrate can be written respectively as:

$$
\begin{gathered}
\sigma_{\text {water/ice }} \cos \theta_{\mathrm{n}}+\sigma_{\text {silica/ice }}=\sigma_{\text {silica/water }} \\
\sigma_{\text {ice/air }} \cos \theta_{\text {ice/air }}+\sigma_{\text {silica/ice }}=\sigma_{\text {silica/air }} \\
\sigma_{\text {water/air }} \cos \theta_{\text {water/air }}+\sigma_{\text {silica/water }}=\sigma_{\text {silica/air }}
\end{gathered}
$$
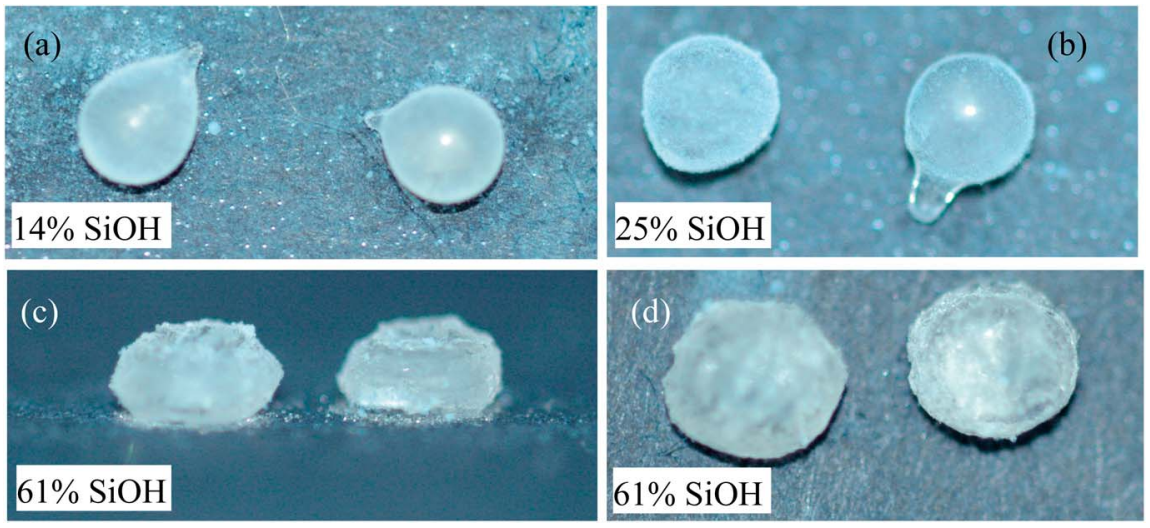

Fig. 3 Final shapes of liquid marbles $(20 \mu \mathrm{L})$ solidified in a freezer at a temperature of $\sim-15^{\circ} \mathrm{C}$. Protrusions appear for the marbles coated with the more hydrophobic particles, (a) $14 \% \mathrm{SiOH}$ and (b) $25 \% \mathrm{SiOH}$. Lateral expansion occurs for the marbles coated with the least hydrophobic particles $(61 \% \mathrm{SiOH})$, (c) side view and (d) top view. 
Therefore,

$$
\cos \theta_{\mathrm{n}}=\frac{\sigma_{\text {ice } / \text { air }} \cos \theta_{\text {ice } / \text { air }}-\sigma_{\text {water/air }} \cos \theta_{\text {water } / \text { air }}}{\sigma_{\text {water } / \text { ice }}}
$$

where $\sigma$ is the interfacial energy between the different phases.

Referring to eqn (6), values of $\sigma_{\text {ice/air }}, \sigma_{\text {water/air }}$ and $\sigma_{\text {water/ice }}$ exist in the literature. The contact angle of water in air at room temperature, $\theta_{\text {water/air, }}$, on substrates composed of compressed fumed silica particles has been measured by Kostakis et al., ${ }^{23}$ and it decreases progressively with increasing $\mathrm{SiOH}$ content (or particle hydrophilicity), as shown in Fig. 4. This is because the particles adsorbed at the air-water interface have different immersion depths in water depending on their hydrophobicity (inset in Fig. 4).

In order to estimate $\theta_{\mathrm{n}}$, it is necessary to measure the contact angle of an ice 'drop' in air $\left(\theta_{\text {ice/air }}\right)$ on a silica substrate obtained by freezing a water droplet $(2 \mu \mathrm{L})$ on the compressed silica discs. In order to make this meaningful, all the measurements were performed at a fixed temperature close to $0{ }^{\circ} \mathrm{C}\left(\sim-5^{\circ} \mathrm{C}\right) . \theta_{\text {water/air }}$ has also been measured at this temperature just before freezing. The two sets of contact angles obtained are listed in Table 1 for five substrate discs differing in the hydrophobicity of the silica particles. The values of $\theta_{\text {water/air }}$ in the present study are somewhat lower than the data given in Fig. 4 although they show a similar decrease with increasing $\mathrm{SiOH}$ content. This suggests that the temperature also plays a role in determining the contact angles that particles exhibit with interfaces. The interfacial energies $\sigma_{\text {ice/air }}, \sigma_{\text {water/ice }}$ and $\sigma_{\text {water/air }}$ at $0{ }^{\circ} \mathrm{C}$ are 104, 28 and $76 \mathrm{~mJ} \mathrm{~m}^{-2}$, respectively. ${ }^{24}$ As a result, $\theta_{\mathrm{n}}$ can be calculated using eqn (6) with values being presented in Table 1 . Values of $\theta_{\mathrm{n}}$ decrease with decreasing particle hydrophobicity, implying that more hydrophilic particles possess better ability to lower the nucleation barrier (having smaller $f\left(\theta_{\mathrm{n}}\right)$ ).

In addition to the intrinsic surface chemistry of the particles, the different immersion depths of particles at the air-water

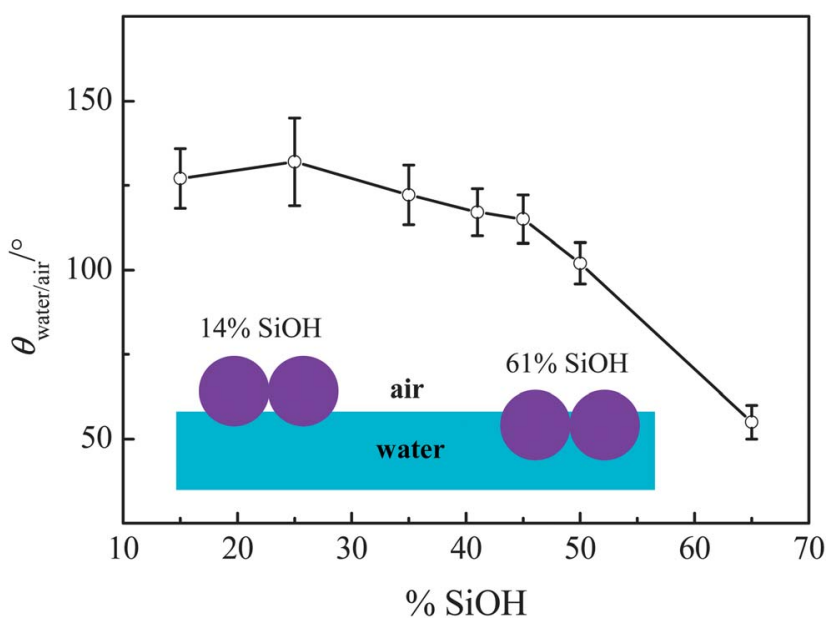

Fig. 4 Advancing contact angles of water in air (room temperature) on discs of compressed fumed silica particles versus particle hydrophobicity. Re-drawn from ref. 23. The sketch indicates the position of particles at the air-water interface for very hydrophobic and the least hydrophobic particles.
Table 1 Average contact angles of water and ice 'drops' in air on discs of compressed fumed silica particles of different hydrophobicities (measured at $\approx-5{ }^{\circ} \mathrm{C}$ through water or ice in degrees) and calculated values of $\theta_{\mathrm{n}}$ (ice 'drop' in water in degrees) using eqn (6). The measurement error is less than $1.5^{\circ}$

\begin{tabular}{lrrrrr}
\hline \multicolumn{5}{c}{ SiOH content on the particle surface } \\
\cline { 2 - 6 } & $14 \%$ & $25 \%$ & $36 \%$ & $50 \%$ & $61 \%$ \\
\hline$\theta_{\text {water/air }}$ & 110.4 & 97.1 & 94.5 & 51.4 & 35.2 \\
$\theta_{\text {ice/air }}$ & 99.1 & 86.2 & 82.1 & 47.3 & 31.2 \\
$\theta_{\mathrm{n}}$ & 69.0 & 54.4 & 43.7 & 34.4 & 16.4
\end{tabular}

interface change the effective solid surface area and the interface geometry which induce ice nucleation. A larger area immersed in water $(61 \% \mathrm{SiOH})$ may have a stronger catalytic effect on ice nucleation compared with particles exposing a large surface area to air. Furthermore, for the very hydrophobic particles $(14 \% \mathrm{SiOH})$, more than half of the particle surface is exposed to air. Nucleation occurs on a convex surface because the water-particle interface is in the shape of a spherical cap. In contrast, for the least hydrophobic particles $(61 \% \mathrm{SiOH})$, they are trapped at the air-water interface with more than half immersed in water. Because of this we hypothesise that water can penetrate the cavities formed between particles within the marble. For a given $\theta_{\mathrm{n}}$, nucleation occurs most readily on concave substrates compared with that on a flat surface. ${ }^{25-27}$ Thus, it can be expected that for the least hydrophobic particles the concave cavities between adjacent particles prefer to act as the nucleation sites. It is worth noting that the bare air-water interface is not expected to act as the preferred nucleation site.

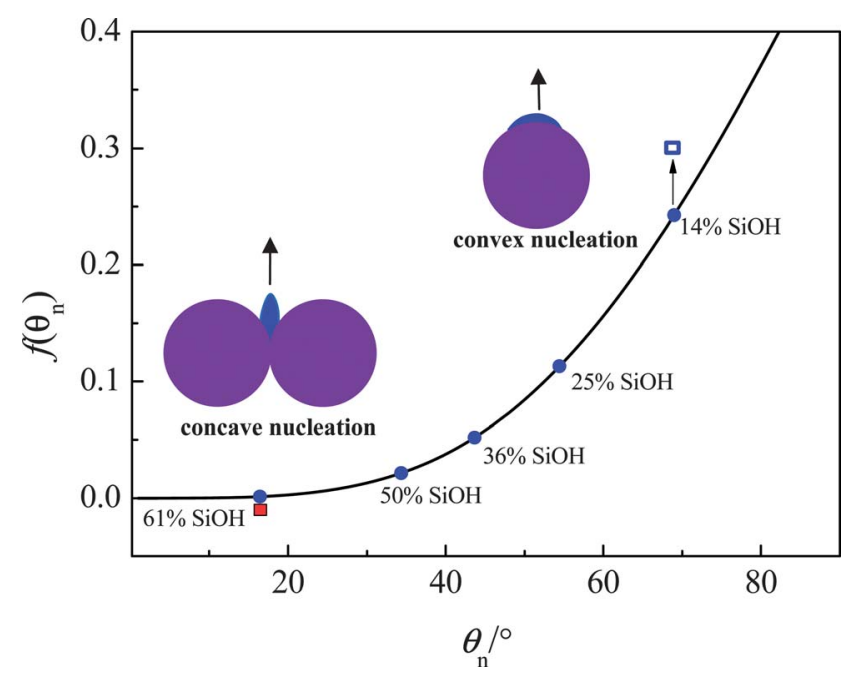

Fig. 5 Effect of the surface curvature of a solid on $f\left(\theta_{n}\right)$. The full curve represents $f\left(\theta_{\mathrm{n}}\right)$ against $\theta_{\mathrm{n}}$ for a planar substrate. The filled circles are obtained using the data in Table 1 . The points for $14 \%$ (unfilled square) and $61 \% \mathrm{SiOH}$ (filled square) particle surfaces are assumed to lie above and below the curve respectively because of the corresponding convex and concave nucleation. Due to both the convex nucleation and high $\theta_{n}, f\left(\theta_{n}\right)$ for a particle possessing $14 \% \mathrm{SiOH}$ is much higher than that for one possessing $61 \% \mathrm{SiOH}$ for which nucleation occurs in the concave cavities between particles. In the inset images, dark blue represents the ice embryo, purple represents the particles. 
(a)

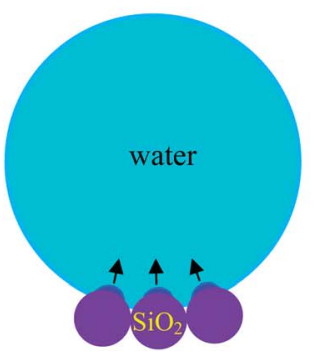

(b)

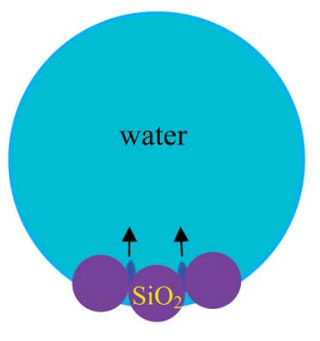

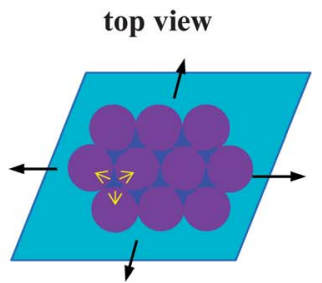

Fig. 6 Different nucleation sites in liquid water (light blue) marbles coated with particles of different hydrophobicities (purple). (a) For the marbles coated with very hydrophobic particles $(14 \% \mathrm{SiOH}$ ), ice nucleation (dark blue) starts from the convex surface of silica particles. (b) For the marbles coated with the least hydrophobic particles $(61 \% \mathrm{SiOH})$, embryos tend to form in the concave cavities between silica particles. The continuous nucleation and volume expansion caused by freezing lead to lateral stretching of the silica particle network.

In order to reach the same critical nucleus radius, $r^{*}$, the different nucleation sites lead to different energy barriers. In general, $r^{*}=-2 \sigma_{\text {water/ice }} / \Delta G_{\mathrm{m}}$, in which $\Delta G_{\mathrm{m}}$ is proportional to the degree of undercooling. ${ }^{22}$ For undercooling at $15{ }^{\circ} \mathrm{C}$, (the corresponding freezing temperature of $-15{ }^{\circ} \mathrm{C}$ for experiments in Fig. 1-3), $r^{*} \approx 6 \mathrm{~nm}$ (ref. 28). This indicates that the embryo size is much smaller than the particle aggregates which are around $150 \mathrm{~nm}$ in diameter. ${ }^{29,30}$ Compared with a planar substrate, the energy barrier is larger for a convex substrate whilst it is smaller for a concave one. ${ }^{31}$ This implies that, at the same degree of undercooling, $f\left(\theta_{\mathrm{n}}\right)$ for the $61 \% \mathrm{SiOH}$ particles should be much lower than that of the $14 \% \mathrm{SiOH}$ particles, as shown in Fig. 5.

From the above analysis, we propose the following freezing scenarios for the marbles, as illustrated in Fig. 6. For the marbles coated with the very hydrophobic particles $(14 \% \mathrm{SiOH}$, Fig. 6(a)), heterogeneous nucleation starts on the convex surface of the particles which are in contact with or very close to the cold plate. Subsequently ice crystals grow into the droplet. Due to the relatively large value of $f\left(\theta_{n}\right)$, nucleation on particles far from the bottom of the marble is quite difficult to attain. This is similar to the case of a pure water droplet freezing on a superhydrophobic surface where heterogeneous nucleation is only induced by the substrate. As a result they exhibit a similar final frozen shape, as shown in Fig. 1(a) and (b). In contrast, for marbles coated with the least hydrophobic particles $(61 \% \mathrm{SiOH}$, Fig. 6(b)), heterogeneous embryos emerge from the concave cavities in between the particles. ${ }^{25,26}$ Due to the very low magnitude of $f\left(\theta_{\mathrm{n}}\right)$ (close to 0 ), continuous nucleation events may take place. It should be noted that the particles form a network structure at the air-water interface as a result of the partial fusion of the primary particles. ${ }^{32}$ The volume expansion of the growing ice embryos in the cavities (meshes) may stretch the particle network, as illustrated in Fig. 6(b) top view, leading to the laterally inflated shape of the frozen marble.

In summary, we have observed interesting and recoverable shape transitions of liquid water marbles during freezing and re-melting cycles which strongly depend on the hydrophobicity of the particles used to coat them. In addition to the possible Marangoni convection induced by the temperature gradient, ${ }^{16}$ we propose that the Gibbs free energy barrier and the site of heterogeneous nucleation depend on the particle wettability and play an important role. Relatively hydrophilic particles greatly reduce the energy barrier for nucleation and lead to ice nucleation in cavities between the particles. Therefore, continuous nucleation occurs resulting in the lateral stretching of the particle shell. Our results reveal that the catalytic effect of a particle surface on heterogeneous nucleation depends on the position of the particles at the air-water interface, determined partly by their surface chemistry. Various shapes of the frozen liquid marbles can be obtained by tuning the hydrophobicity of the particles coating the marbles. This finding may be utilized in the fabrication of functional particles with different shapes.

\section{Acknowledgements}

This work was supported by the National Natural Science Foundation of China (Grant no. 51301139), Shaanxi Provincial Natural Science Foundation (Grant no. 2012JQ1016), NPU Foundation for Fundamental Research (JCY20130147, JC201125 and JC201153) and Specialized Research Fund for the Doctoral Program of Higher Education (Grant no. 20126102120058). We thank Dr Quanwen Hou and Mr Delu Geng for helpful discussions. We also thank Wacker-Chemie, Burghausen for the fumed silica powders.

\section{References}

1 E. Bormashenko, Curr. Opin. Colloid Interface Sci., 2011, 16, 266.

2 G. McHale and M. I. Newton, Soft Matter, 2011, 7, 5473.

3 D. Quéré and P. Aussillous, Proc. R. Soc. London, Ser. A, 2006, 462, 973.

4 T. H. Nguyen, K. Hapgood and W. Shen, Chem. Eng. J., 2010, $162,396$.

5 L. Gao and T. J. McCarthy, Langmuir, 2007, 23, 10445.

6 A. Lafuma and Quéré, Nat. Mater., 2003, 2, 457.

7 P. S. Bhosale, M. V. Panchagnula and H. A. Stretz, Appl. Phys. Lett., 2008, 93, 034109.

8 P. Aussillous and D. Quéré, Nature, 2001, 411, 924. 
9 Y. Zhao, J. Fang, H. Wang, X. Wang and T. Lin, Adv. Mater., 2010, 22, 707.

10 T. Arbatan, L. Li, J. Tian and W. Shen, Adv. Healthcare Mater., 2012, 1, 80.

11 Y. Xue, H. Wang, Y. Zhao, L. Dai, L. Feng, X. Wang and T. Lin, Adv. Mater., 2010, 22, 4814.

12 E. Bormashenko, R. Pogreb, T. Stein, G. Whyman, M. Schiffer and D. Aurbach, J. Adhes. Sci. Technol., 2011, 25, 1371 .

13 L. Zhang, D. Cha and P. Wang, Adv. Mater., 2012, 24, 4756.

14 E. Bormashenko, R. Pogreb, T. Stein, G. Whyman and M. Hakham-Itzhaq, Appl. Phys. Lett., 2009, 95, 264102.

15 M. I. Newton, D. L. Herbertson, S. J. Elliott, N. J. Shirtcliffe and G. McHale, J. Phys. D: Appl. Phys., 2007, 40, 20.

16 A. Hashmi, A. Strauss and J. Xu, Langmuir, 2012, 28, 10324.

17 D. Y. Zang, Z. Chen, Y. J. Zhang, K. J. Lin, X. G. Geng and B. P. Binks, Soft Matter, 2013, 9, 5067.

18 D. Y. Zang, F. Li, X. G. Geng, K. J. Lin and P. S. Clegg, Eur. Phys. J. E, 2013, 36, 59.

19 J. Wang, Z. Liu, Y. Gou, X. Zhang and S. Cheng, Sci. China, Ser. E: Technol. Sci., 2006, 49, 590.
20 L. Huang, Z. Liu, Y. Liu, Y. Gou and L. Wang, Exp. Therm. Fluid Sci., 2012, 40, 74.

21 Z. Jin, Q. Dong, S. Jin and Z. Yang, Int. Conf. Fluid Dynamics \& Thermodyn. Technol., 2012, vol. 33, p. 125.

22 W. Kurz and D. J. Fisher, Fundamentals of Solidification, Trans Tech Publications, Aedermannsdorf, 1st edn, 1984, p. 27.

23 T. Kostakis, R. Ettelaie and B. S. Murray, Langmuir, 2006, 22, 1273.

24 H. R. Pruppacher and J. D. Klett, Microphysics of clouds and precipitation, Kluwer, Netherlands, 1997, p. 160.

25 M. Qian, Acta Mater., 2007, 55, 943.

26 D. Turnbull, J. Chem. Phys., 1950, 18, 198.

27 N. H. Fletcher, J. Atmos. Sci., 1969, 26, 1266.

28 X. Y. Liu and N. Du, J. Biol. Chem., 2004, 279, 6124.

29 D. Y. Zang, A. Stocco, D. Langevin, B. Wei and B. P. Binks, Phys. Chem. Chem. Phys., 2009, 11, 9522.

30 D. Y. Zang and Y. J. Zhang, Acta Phys. Sin., 2012, 61, 026803.

31 B. Chalmers, Principles of Solidification, John Wiley, New York, 1964, pp. 77-83.

32 B. P. Binks, Curr. Opin. Colloid Interface Sci., 2002, 7, 21. 